\title{
Surface Engineering for Energy Sustainability and Bio-applications
}

Sandip P. Harimkar, Arvind Agarwal, Sudipta Seal, and Narendra Dahotre

Surfaces and interfaces are expected to play an important role in determining the efficiency, structural integrity, and reliability of various systems, including energy generation/storage devices, machinery elements, and bioimplants. Surface engineering is vital for tailoring the composition, microstructure, texture of the surfaces at multiple length scales to improve the properties. The forefront of this area continues to evolve with exploration of new concepts and methodologies, novel surface processing routes, microstructure analysis, property evaluations, and simulation/modeling. The feature topic of this JOM issue is intended to provide the readers a representative spectrum of current research efforts in this rapidly evolving field of surface engineering with special emphasis on energy systems and bioapplications. The papers are discussed briefly.

The first paper, "Application of Carbon Nanostructures-Energy to Electronics," by I. Lahiri et al., presents an overview of the development of new engineering nanomaterials (CVD grown graphene and carbon nanotubes) and their applications in the field of energy generation and storage. The proposed carbon-based materials are also attractive for electronics, sensors, biotechnology, energy, and structural applications.

The second paper, "Development and Surface Characterization of Novel Alumina-based Composites for Energy Efficient Sliding Systems," by R. Paluri and S. Ingole, presents the results of their investigations on development of self-lubricating materials with superior surface properties for reduced friction and wear applications. The paper also provides an overview of the recent developments in friction, wear, and lubrication processes for improved performance and energy efficiency.

The third paper, "Microstamped ECM Proteins Enhance Endothelial Cell Adhesion and Directed Growth within a Bioassay Chamber," by D. Rubenstein and M. Frame, presents detailed investigations on microvascular endothelial cell growth on microstamped patterns of extracellular matrix proteins $(\mathrm{ECM})$. A combination of photo- and soft-lithography was used to pattern micro-scale features.

The fourth paper, "Laser Surface Modification of Metallic Biomaterials," by A. Bandyopadhyay et al., provides an overview of laser surface modification of Ti/Ti-6Al-4V alloy with and without functional gradation in composition and their microstructure. The proposed laser surface modifications are expected to improve the wear and biological properties for load bearing orthopedic applications.

We would like to thank the authors for their excellent contributions to this issue of JOM. We hope that the articles will possibly trigger new thoughts and discussions for future developments.

We take this opportunity to thank all the presenters and audience who participated in our symposium Surfaces and Heterostructures at Nano-or-Micro Scale and their Characterization in San Diego, during The TMS 2011 Annual Meeting in February 2011. This symposium was co-organized by the Surface Engineering and the Nanomaterials committees of TMS. The Surface Engineering Committee will be organizing the symposium Advances in Surface Engineering: Alloyed and Composite Coatings at the TMS 2012 Annual Meeting. (A complete list of symposia planned for the TMS 2012 Annual Meeting and Exhibition can be found in News \& Update on page 3.)

Drs. Harimkar (Oklahoma State University), Agarwal (Florida International University), Seal (University of Central Florida), and Dahotre (University of North Texas) are the JOM advisors from the MPMD's Surface Engineering Committee.

\section{Sandip P. Harimkar, Arvind Agarwal, Sudipta Seal, and Narendra Dahotre are TMS Members!}

To read more about them, turn to page 10. To join TMS, visit www.tms.org/Society/Membership.aspx. 